\title{
Ueber die Einwirkung des Broms auf Trimethylen unter verschiedenen Bedingungen; \\ von
}

G. Gustavson.

Durch die Anwesenheit mancher Körper wird die Reaction zwischen Trimethylen und Brom sehr beschleunigt. In dieser Richtung wirken nicht nur solche Körper, wie Aluminiumbromid und -chlorid, Ferrichlorid, Zinkchlorid, Jod, sondern auch einige Brommetalle in wässriger Lösung, sowie auch Bromwasserstoffsäure. Diese Eigenschaft der Bromwasserstoffsäure hat besonders meine Aufmerksamkeit in Anspruch genommen. Indem ich die Reaction zwischen Brom und Trimethylen unter verschiedenen Bedingungen studirte, habe ich bemerkt, dass scharf getrocknetes Trimethylen und Brom nur sehr träge auf einander einwirken; einige Tropfen Wasser befördern aber die Reaction merklich. Man konnte vermuthen, dass eigentlich nicht Wasser, sondern Bromwasserstoffsäure auf die Reaction beschleunigend wirkte, weil das Wasser nach der Reaction immer Bromwasserstoff enthielt. Auf solche Weise kam ich dazu, die Einwirkung der Bromwasserstoffsäure während der Reaction zwischen Brom und Trimethylen zu versuchen. Wenn man Brom mit sehr kleinen Quantitäten von Bromwasserstoffsäure $\left(\mathrm{HBr}+5 \mathrm{H}_{2} \mathrm{O}\right)$ mischt, z. B. auf $12 \mathrm{Grm}$. Brom 0,3 Ccm. Bromwasserstoffsäure, und in diese Mischung einen Strom von Trimethylen leitet, so beobachtet man, dass das Trimethylen von Brom unter starker Erwärmung des letzteren und ebenso vollständig wie Propylen absorbirt wird. ${ }^{1}$ ) Die Energie der Reaction nimmt aber bald ab und Brom lässt später einen Theil von Trimethylen durchgehen. Die Ursachen dieser Erscheinung, welche später näher erörtert werden, liegen nicht in einer Ausnutzung von Bromwasserstoffsäure. Dieselbe

1) Die Reaction gelingt nur mit ganz chlorfreiem Brom. Um das Brom zu reinigen, schüttelt man dasselbe mit Bromwasserstoffsäure $\left(\mathrm{HBr}+5 \mathrm{H}_{2} \mathrm{O}\right)$, wäscht mit Wasser, trocknet und destillirt, das bei $59^{\circ}$ bis $60^{\circ}$ Uebergehende auffangend.

Journal f. prakt. Chemie [2] Bd. 62 . 
274 Gustavson: Ueb.d. Einw. d. Broms auf Trimethylen etc.

bleibt immer und nimmt sogar am Ende der Reaction zu. Andere Reactionsprodukte sind: Trimethylenbromid, Propylenbromid, deren weiteren Bromirungsprodukte und kleine Mengen von Propylbromid.

In der folgenden Tabelle sind die Versuche zusammengestellt, die ausgeführt wurden, um den Einfluss der Concentration und der Menge der zum Brom zugefügten Bromwasserstoffsäure auf den Process zu bestimmen, sowie einige andere sich hieran schliessende Versuche.

\begin{tabular}{|c|c|c|c|c|c|c|c|c|}
\hline 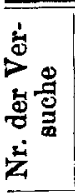 & 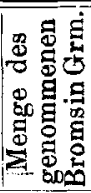 & 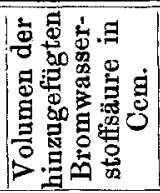 & 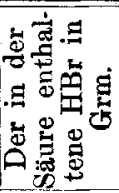 & 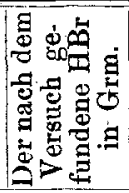 & 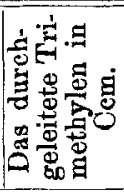 & 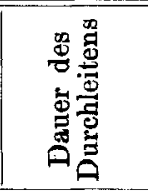 & 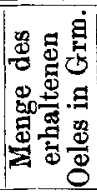 & 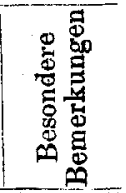 \\
\hline$I$ & 12 & - & - & 0,0189 & 2000 & 1 St. $30 \mathrm{M}$. & 0,7 & \\
\hline II & 12 & - & - & 0,010 & 1750 & 1,30, & 0,85 & bei $60^{\circ}$ \\
\hline III & 12 & - & - & 0,0819 & 2000 & $1,30 \%$ & 1,75 & $\begin{array}{l}\text { Es waren } \\
0,3 \text { Ccm. } \\
\mathrm{H}_{2} \mathrm{O} \text { hin- } \\
\text { zugefügt }\end{array}$ \\
\hline IV & 12 & 0,3 & 0,082 & 0,246 & 1750 & 1,30, & 3,25 & \\
\hline V & 12 & 0,3 & 0,215 & 0,251 & 1750 & $1,30 \%$ & 4,00 & \\
\hline VI & 12 & 1,0 & 0,694 & 0,6901 & 1750 & 1,30, & 3,60 & \\
\hline VII & 12 & 2,0 & 1,388 & $\begin{array}{l}\text { Nicht be- } \\
\text { stimmt }\end{array}$ & 1750 & $1 " 30 "$ & 2,90 & \\
\hline VIII & 12 & 3,0 & 2,082 & 1,774 & 1750 & $1,50 "$ & 2,65 & \\
\hline IX & 12 & 0,13 & 0,0868 & 0,1253 & 1750 & $1,50 \%$ & 3,4 & \\
\hline $\mathbf{X}$ & 12 & 1 Tropfen & 0,0144 & 0,0468 & 1750 & $1,50 "$ & 1,3 & \\
\hline XI & 12 & 0,3 & 0,215 & $\begin{array}{l}\text { Nicht be- } \\
\text { stimmt }\end{array}$ & 1750 & $1 " 15 "$ & 3,9 & \\
\hline
\end{tabular}

Aus den angeführten Versuchen ist ersichtlich, 1. dass man, um die grösste Ausbeute von Bromiden aus Trimethylen zu erhalten, zu 12 Grm. Brom 0,1-1,0 Ccm. Bromwasserstoffsäure $\left(\mathrm{HBr}+5 \mathrm{H}_{2} \mathrm{O}\right)$ hinzufügen muss. Die vortheilhafteste Menge befindet sich übrigens in der Mitte zwischen diesen Zahlen und ist jedenfalls nicht weit von $0,3 \mathrm{Ccm}$. (für $12 \mathrm{Grm}$. Br) entfernt, welche Menge ich auch nach den ersten Versuchen beibehalten habe. 2. Benutzt man anstatt der Säure $\mathrm{HBr}+5 \mathrm{H}_{2} \mathrm{O}$ das gleiche Volumen Säure geringerer Concentration, so wird die Einwirkung des Broms schwächer. 3. Nimmt man anstatt schwacher Säure das gleiche Volumen Wasser, 
so vermindert sich die Einwirkung des Broms noch mehr. 4. Lässt man jedoch das Wasser ganz weg, so erweist sich die Einwirkung als noch schwächer.

Die in der Tabelle angeführten Versuche sind mittelst Durchleitens von Trimethylen ${ }^{1}$ ) durch Brom ausgeführt. Es sei bemerkt, dass beim Durchleiten von Trimethylen durch Lösungen von Brom in Bromwasserstoffsäure sich diese in zwei nicht mischbare Schichten theilen, von welchen die obere, wässrige, fast die ganze Bromwasserstoffsäure enthält. Das Brom jedoch und die Produkte der Einwirkung von Brom auf Trimethylen sind so auf die beiden Schichten vertheilt, dass sich die Hauptmasse der Bromide in der unteren, die Farbe von Brom zeigenden Schicht befindet. Es wurde ferner beobachtet, dass beim fortgesetzten Leiten von Trimethylen durch die Schichten die untere sich vergrösserte, die obere aber sich verringerte. Mit dieser Veränderung der Volume macht sich eine Abnahme der Reactionsenergie durch Sinken der Temperatur der Mischung, wie durch bedeutend geringere Absorption des durchgeleiteten Trimethylens bemerkbar.

Ueber den Verlauf des Absorptionsprocesses in Gegenwart von Bromwasserstoffsäure kann man sich aus folgenden Versuchen eine Vorstellung machen:

XII. Gemischt wurden 24 Grm. Brom und 0,4316 Grm. $\mathrm{HBr}$ in Form von Bromwasserstoffsäure $\mathrm{HBr}+5 \mathrm{H}_{2} \mathrm{O}$ (ungefähr 0,6 Ccm.). In Folge der durch Brom verursachten dunklen Färbung der beiden Schichten sind dieselben nur schwer $\mathrm{zu}$ unterscheiden. Im Ganzen wurden 2 Liter Trimethylen durchgeleitet, jedes Liter in 40 Min., aber nur die ersten $650 \mathrm{Ccm}$. wurden vollständig vom Brom absorbirt; über die Hälfte des zweiten Liters blieb unabsorbirt. Hierbei sank auch die Temperatur des Broms. Nach dem Versuche bestand die Flüssigkeit aus zwei Schichten; die untere war viel stärker von Brom gefärbt, als die obere, deren Volumen

1) Die Gewinnung und die Reinigung des Trimethylens wurden auf die Weise in einer Operation vereinigt, dass man das nach meiner $\mathrm{Me}$ thode direct aus dem, käuflichen Trimethylenbromid erhaltene Gas vor dem Eintritt in den Gasometer durch trocknes Brom und dann durch Natronlauge und durch ein Controlrohr mit verdünnter Permanganatlösung leitete. 
276 Gustavson: Ueb.d. Einw. d. Broms auf Trimethylen etc.

sich verringert hatte. Die ganze Flüssigkeit wurde mit Wasser bearbeitet, und im Ganzen wurden mit dem im zweiten Gefäss vom Wasser zurückgehaltenen $0,5523 \mathrm{Grm}$. $\mathrm{HBr}$ gefunden. Die nach dem Waschen mit Wasser gebliebene, homogene, stark durch Brom gefärbte Flüssigkeit (die untere Schicht) wurde in zwei Theile von je 11,5 Grm. getheilt. Aus dem einen wurde durch Bearbeitung mit Natronlauge ein Oel ausgeschieden, dessen Gewicht 4,15 Grm. betrug. Der andere Theil wurde mit $0,3 \mathrm{Ccm}$. Bromwasserstoffsäure, $\mathrm{BBr}+5 \mathrm{H}_{2} \mathrm{O}$, versetzt und langsam ein Liter Trimethylen durchgeleitet, wonach aus dieser Portion $5 \mathrm{Grm}$. Oel gewonnen wurden. Folg. lich fährt das Trimethylen fort, mit Brom zu reagiren, jedoch unvergleichlich langsamer, als zu Beginn des Versuches, wo das Gas vollständig und mit starker Wärmeentwicklung vom Brom absorbirt wurde.

XIII. Zur Bestimmung des Erhitzungsgrades des Broms während der ersten Periode der Reaction, wurden $12 \mathrm{Grm}$. Brom und $0,3 \mathrm{Ccm}$. $\mathrm{HBr}+5 \mathrm{H}_{2} \mathrm{O}$ mit einem kleinen Thermometer zusammen in den Absorptionsapparat, ein mit eingeschliffenem Stöpsel mit Röhren versehenes Reagensglas, gethan. In $20 \mathrm{Min}$. wurden $225 \mathrm{Ccm}$. Trimethylen hindurchgeleitet. Bei einer Aussentemperatur von $18^{\circ}$ erhitzte sich das Brom bis $47^{\circ}$. In der Messröhre hatten sich $12,5 \mathrm{Ccm}$. unabsorbirtes Gas gesammelt, welches beim Anzünden nicht auflammte. An Oel wurde 1,3 Grm. gewonnen.

XIV. Der Versuch wurde wiederholt zwecks Lösung der Frage: bleibt während der ersten Periode des Durchleitens von Trimethylen durch Brom und Bromwasserstoffsäure brennbares Gas unabsorbirt oder nicht? $\mathrm{Zu}$ diesem $\mathbf{Z}$ weck wurde das Luftvolumen in den mit Brom und mit Natronlauge gefüllten Apparaten bis auf ein Minimum beschränkt und vor dem Versuche durch die Trockenröhren Trimethylen! längere Zeit geleitet. Durch $20 \mathrm{Grm}$. Brom und 0,5 Ccm. $\mathrm{HBr}+$ $5 \mathrm{H}_{2} \mathrm{O}$ wurden in einer halben Stunde $500 \mathrm{Ccm}$. Trimethylen geleitet. In der Messröhre hatten sich $8 \mathrm{Ccm}$. Gas gesammelt, welches nicht aufflammte. An Oel wurden 2,9 Grm. gewonnen.

Folglich wird zu Beginn des Durchleitens das Trimethylen vollständig von den besagten Mischungen von Brom 
und Bromwasserstoffsäure absorbirt. Unabsorbirt entweicht nur Luft.

Als ich zu Anfang dieses Jahres mit den oben angeführten Versuchen beschäftigt war, erschien eine Abhandlung von K. Auwers und O. Anselmino ${ }^{\mathrm{I}}$ ), aus welcher hervorging, dass ein Gemisch von Brom und Bromwasserstoffsäure die Abspaltung von Methylgruppen aus den bromirten Homologen des Phenols bewirkt. So z. B. entsteht durch Einwirkung dieses Gemisches bei Zimmertemperatur aus Dibrompseudocumenol, $\mathrm{C}_{8}\left(\mathrm{CH}_{3}\right)_{3} \mathrm{Br}_{2} \mathrm{OH}$,Tribromxylenol, $\mathrm{C}_{6}\left(\mathrm{CH}_{3}\right)_{2} \mathrm{Br}_{3} \mathrm{OH}$. Es hat hier folglich eine der seltenen, tiefgreifenden Reactionen stattgefunden, die sonst nur durch so stark wirkende Reagentien, wie Antimonpentachlorid, Chlor- oder Bromaluminium, hervorgerufen werden, oder die nur bei sehr hoher Temperatur vor sich gehen. Bemerkenswerth ist, dass der Bromwasserstoff bei diesen Reactionen unbedingt in wässriger Lösung verwandt werden muss: wasserfreier Bromwasserstoff und Brom sind nicht im Stande, besagte Abspaltung sogar bei einer Temperatur von über $100^{\circ}$ hervorzurufen.

Obgleich die Untersuchungen von Auwers und Anselmino auf einem ganz anderen Gebiete liegen wie die meinigen, so findet sich doch bei den einen wie bei den anderen in erster Linie die bisher nicht bemerkte Abhängigkeit der Einwirkungsenergie des Broms auf einige organische Verbindungen von der Gegenwart der Bromwasserstoffsäure. Ich nehme an, dass die beobachtete Abhängigkeit durch die Entstehung einer Verbindung (möglicher Weise auch mehrerer Verbindungen) von Brom und Bromwasserstoff bedingt wird. Es reagirt demnach nicht freies Brom, sondern das Brom der sich bildenden Verbindungen $\left(\mathrm{HBr}_{3}, \mathrm{HBr}_{5} \ldots.\right)$, und die Rolle des Bromwasserstoffs in diesen Reactionen ist in ihren Hauptzügen analog der von Aluminiumbromid und einigen anderen die Reactionen organischer Verbindungen fördernden Substanzen. ${ }^{2}$ ) Der Unterschied besteht darin, dass in dem zu betrachtenden Falle die Substanz nicht die Activität der organischen Verbindung erhöht, indem sie sich mit ihr verbindet, sondern die

1) Ber, 32, 3587 ,

2) M. Nencki, Ber. 32, 2414. 
des Broms, und zwar auch dadurch, dass sie eine Verbindung mit ihm eingeht. Das ändert jedoch die Sache nicht, und in dieser Hinsicht kann man den Bromwasserstoff in wässriger Lösung zu den übrigen Reactionserregern zählen. Er wirkt nach Art der Fermente, indem er nach und nach die mit ihm verbundene Substanz abgiebt und sie so allmäblich in die Reaction einfuhrt; er selbst bleibt aber zuletzt in Form und Gewicht unverändert zurück, soweit dies nicht durch Nebenreactionen verhindert wird.

Wenn die ausgesprochene Voraussetzung richtig ist, so muss die Energie der Reaction sich in directer Abbängigkeit von Bedingungen befinden, die eine reichliche Bildung und schnelle Erneuerung von Verbindungen zwischen Brom und Bromwasserstoffsäure erlauben. Dass eine solche Abhängigkeit vorhanden sei, stellte sich bei der Untersuchung von Ursachen der Reactionverlangsamung heraus. Diese Ursachen liegen nicht am Mangel an Bromwasserstoffsäure, oder ain Brommangel, sondern darin, dass sich diese Substanzen in verschiedenen Schichten concentriren. Wenn man durch eine gesättigte Lösung von Brom in Bromwasserstoffsäure, in der sich folglich Verbindungen dieser beiden Körper befinden, Trimethylen leitet, so theilt sich die Flüssigkeit in Folge der Bildung von Bromiden und der Anwesenheit von Wasser in zwei Schichten. Hiermit wird der Anfang gemacht zur Scheidung der Bromwasserstoffsäure (die obere wässrige Schicht) vom Brom (untere Schicht). Die Bedeutung dieser Erscheinung besteht darin, dass ein Theil des Broms, welcher, vermischt mit der Bromwasserstoffsäure, durch seine Anwesenheit die Dissociation der Verbindungen $\mathrm{HBr}_{3}$ u. s. w. beschränkt, jetzt sich in der unteren Schicht concentrirt. Je weiter der Process vorschreitet, eine um so grössere Menge von Bromiden bildet sich, und um so mehr freies Brom tritt aus der oberen Schicht (in der die Bromwasserstoffsäure zurückbleibt) in die untere. Die Dissociation der Verbindungen von Brom und Bromwasserstoffsäure wächst und parallel hiermit nimmt die Energie der Reaction ab. $\mathrm{Zu}$ dieser Ursache der Verlangsamung der Reaction tritt noch eine andere: das Trimethylen reagirt mit den restirenden Verbindungen von Brom und Bromwasserstoffsäure und zerstört sie. Ihre Entstehung 
Gustavson: Ueb.d. Einw.d. Broms auf Trimethylen etc. 279

wird dadurch sehr gehemmt, dass sich das Brom in der anderen Schicht befindet. Wir haben es mit der Störung des Gleichgewichts zwischen zwei flüssigen Phasen zu thun: die Wiederherstellung des gestörten Gleichgewichts erfordert in solchen Fällen Zeit.

Man kommt zu demselben Schluss über die Bedeutung der Verbindungen von Brom mit Bromwasserstoffsäure für die Reaction, wenn die Flüssigkeit von Anfang an in zwei Schichten getheilt ist, d. h. wenn ein Ueberschuss von Brom vorhanden ist. Die obere Schicht erscheint dann als gesättigte lösung von Brom in Bromwasserstoffsäure, die untere als ebenfalls gesättigte Lösung von Bromwasserstoffsäure in Brom; diese letzte Schicht besteht aus fast reinem Brom, da Bromwasserstoffsäure sich nur sebr schwer in Brom löst. $\mathrm{Zu} \mathrm{Be-}$ ginn der Reaction geht die Wiederherstellung des durch das Durchleiten des Trimethylens gestörten Gleichgewichts zwischen den zwei Phasen recht rasch vor sich, in Folge des Vorhandenseins der aus Brom bestehenden unteren Schicht. Dieses ist die Periode der erhöhten Einwirkungsenergie des Broms auf Trimethylen, wobei dieses Gas vollständig rom Brom absorbirt wird und die Temperatur der Mischung rasch steigt. Doch in dem Maasse, als die Bildung von Bromiden vorschreitet, verlangsamt sich die Reaction in Folge der Ursachen, welche schon früher betrachtet und erklärt wurden.

Die oben ausgesprochenen Ansichten werden durch die folgenden $\nabla$ ersuche bestätigt.

XV. Die Bestimmung der Löslichkeit von Brom in Bromwasserstoffsäure wurde nach W. F. Alexejew in zugeschmolzenen Röhren ausgeführt: 5,445 Grm. $\mathrm{Br}$ (die Analyse des Broms ergab $100,2 \% \mathrm{Br}$ ) und 0,6404 Grm. $\mathrm{HBr}+5 \mathrm{H}_{2} \mathrm{O}$ (die Säure war durch Destillation gereinigt und enthielt $47,3 \%$ $\mathbf{H B r}$ ) gaben bei Zimmertemperatur homogene Lösung. Beim Erhitzen im Wasserbade wurde das Erscheinen des ersten Tropfens bei $34^{\circ}$ bemerkt, so dass bei dieser Temperatur 100 Thle. Bromwasserstoffsäure 850,2 Thle. Brom lösen, oder die Lösung enthält 4,97\% $\mathrm{HBr}$. Die Löslichkeit des Broms in Bromwasserstoffsäure vermindert sich also bei Erhöhung der Temperatur, - natürlich in Folge der Dissociation der Verbindungen von Bromwasserstoffsäure mit Brom. Ein anderes 
280 Gustavson: Ueb.d.Einw.d. Broms auf Trimethylen ete.

Mal wurde die Löslichkeit bei $18^{\circ}$ durch Schütteln eines Ueberschusses von Brom mit Bromwasserstoffsäure bestimmt; 2,3567 Grm. der oberen Schicht gaben nach Entfernung des Broms durch Erhitzen mit Wasserstoff 0,1145 Grm. HBr, d. h. $4,86 \%$ HBr. Die Bromwasserstoffsäure löst sich in Brom sehr wenig. Brom nimmt bei $18^{\circ}$ nur $0,11 \% \mathrm{HBr}$ in Form von $\mathrm{HBr}+5 \mathrm{H}_{2} \mathrm{O}$ auf. In $14,18 \mathrm{Grm}$. Lösung wurden nur $0,0162 \mathrm{Grm}$. HBr gefunden.

XVI. Um die Veränderungen des Volumens und der Zusammensetzung der Schichten zu bestimmen, wurden folgende Versuche gemacht. In eine $13,4 \mathrm{Grm}$. betragende, bei $18^{\circ}$ gesättigte Lösung von Brom in Bromwasserstoffsäure, was einer Brommenge von $12 \mathrm{Grm}$. entspricht, wurden $1750 \mathrm{Ccm}$. Trimethylen in 1 St. $20 \mathrm{Min}$. hindurchgeleitet. Nach der Operation sind in der Flüssigkeit zwei Schichten bemerkbar. Das Volum der ganzen Flüssigkeit beträgt $5,8 \mathrm{Ccm}$., das der oberen Schicht 1,8 Ccm. Die Analyse der oberen Schicht ergab für 0,6422 $\mathrm{Grm}$. 0,0949 $\mathrm{HBr}$, d. h. $14,7 \% \mathrm{HBr}$ (vor dem Durchleiten des Trimethylens enthielt die homogene Lösung $4,86 \% \mathrm{HBr}$ ). An Oel wurden $3,55 \mathrm{Grm}$. gewonnen.

XVII. Genommen wurden 9,102 Grm. Brom und 0,388 Grm. $\mathrm{HBr}+5 \mathrm{H}_{2} \mathrm{O}$, d. h. das Verhältniss des Broms zur Säure ist dasselbe wie von $12 \mathrm{Grm}$. Brom zu $0,34 \mathrm{Ccm}$. $\mathrm{HBr}+5 \mathrm{H}_{2} \mathrm{O}$. Das Gemisch wurde in einer zugeschmolzenen Röhre bei $18^{\circ}$ geschüttelt und dann in einen engen Messcylinder ausgegossen. Das Volum der ganzen Flüssigkeit betrug $3,2 \mathrm{Ccm}$., das der oberen Schicht 1,3 Ccm. Folglich nimmt bei dem Verhältniss von Brom zu Bromwasserstoffsäure, bei welchem die Absorption des Trimethylens sehr energisch verläuft, die obere Schicht mehr als ein Drittel des ganzen Flüssigkeitsvolums ein. Darauf wurden durch $12 \mathrm{Grm}$. Brom und 0,3 $\mathrm{Ccm}$. Bromwasserstoffsäure ein Liter Trimethylen geleitet. Nach Beendigung des Durchleitens betrug das Volum der Flüssigkeit $3,8 \mathrm{Ccm}$., und das der oberen Schicht hatte sich auf 0,5 Ccm. reducirt. Der Bromwasserstoffgehalt derselben war jedoch stark gestiegen. $0,6463 \mathrm{Grm}$. der oberen Schicht ergaben 0,1009 Grm. HBr, d. h. 15,6\%. An Oel wurden 3 Grm. gewonnen.

XVIII. Zur Entscheidung der Frage, wie gross die Menge der Bromide ist, die beim Durchleiten von Trimethylen durch 
Gustavson: Ueb.d.Einw.d.Broms auf Trimethylen etc. 281

die untere Schicht entstehen, wurde ein besonderer Versuch angestellt. Durch 12 Grm. mit Bromwasserstoffsäure gesättigten und $0,1 \% \mathrm{HBr}$ enthaltenden Broms wurden $1750 \mathrm{Ccm}$. Trimethylen geleitet; es wurden 1,85 Grm. Oel gewonnen. Folglich ergiebt das getrennte Durchleiten durch die obere und die untere Schicht der Combination von $12 \mathrm{Grm}$. Br und $0,3 \mathrm{Ccm} . \mathrm{HBr}+5 \mathrm{H}_{2} \mathrm{O}$ in der Summe eine geringere Menge von Bromiden, als das Durchleiten von Trimethylen durch das Gemisch der beiden Schichten. Hieraus folgt, dass die Rolle der unteren Schicht hauptsächlich in dem Hinzutreten von Brom zu Bromwasserstoffsäure der oberen Schicht besteht (in den ersten Momenten der Reaction), d. h. dass die Reaction durch die Verbindungen der Bromwasserstoffsäure mit Brom bewirkt wird.

XIX. Auf meine Veranlassung wurden in unserem Laboratorium von Frau L. Kaufmann Versuche über die Bromirung von Benzol in Gegenwart von Bromwasserstoffsäure angestellt. Die Versuche wurden bei $25^{0}$ im Thermostat ausgeführt; Dauer des Erhitzens 2 Stunden; das von der Reaction nicht verbrauchte Brom wurde durch Titriren des von ihm ausgeschiedenen Jodes bestimmt. Beim Hinzufügen von Benzol scheidet sich jedoch die Lösung von Brom in Bromwasserstoffsäure sofort in zwei Schichten, was, wie aus dem früher Gesagten hervorgeht, eine erhöhte Dissociation der Verbindungen von Bromwasserstoffsäure mit Brom zur Folge hat. Desswegen sind die folgenden Versuche kaum genügend für eine Schlussfolgerung in dem Sinne, dass die Bromwasserstoffsäure auf die Reaction zwischen Brom und Benzol nicht stark fördernd einwirken kann. Die Versuche ergaben folgende Resultate: 1. Es wurden angewandt $0,5096 \mathrm{Grm} . \mathrm{Br}, 0,2534 \mathrm{Grm} . \mathrm{O}_{6} \mathrm{H}_{6}$ und $0,027 \mathrm{Grm} . \mathrm{HBr}+5 \mathrm{H}_{2} \mathrm{O}$; in die Reaction waren eingetreten 0,0633 Grm. Br, d. h. 12,4\%. 2. 0,4994 Grm. Br, 0,2506 Grm. $\mathrm{C}_{6} \mathrm{H}_{6}$ und $0,027 \mathrm{Grm} . \mathrm{HBr}+5 \mathrm{H}_{2} \mathrm{O}$; in die Reaction waren eingetreten 0,0695 Grm. Br, d. h. 13,9\% 3. 0,9498 Grm. $\mathrm{Br}, 0,4756 \mathrm{Grm} . \mathrm{C}_{6} \mathrm{~B}_{8}$ und $0,0628 \mathrm{Grm} . \mathrm{HBr}+5 \mathrm{H}_{2} 0$; in die Reaction waren eingetreten $0,1167 \mathrm{Grm} . \mathrm{Br}$, d. h. $12,3 \%$. 4. $0,564 \mathrm{Grm}$. Br, 0,2734 Grm. $\mathrm{C}_{6} \mathrm{H}_{6}$ and 0,0484 Grm. $\mathrm{H}_{2} \mathrm{O}$; in die Reaction waren eingetreten $0,0637 \mathrm{Grm}$. Br, d. h. $11,2 \%$. 5. 0,5806 Grm. Br, 0,2796 Grm. $\mathrm{C}_{6} \mathrm{H}_{6}$ und 0,03こ Grm. $\mathrm{H}_{2} \mathrm{O}$; 
282 Gustarson: Ueb.d Einw.d. Broms auf Trimethylen etc.

in die Reaction waren eingetreten $10,3 \%$ Br. 6. 0,5842 Grm. $\mathrm{Br}, 0,2824$ Grm. $\mathrm{C}_{6} \mathrm{H}_{6}$; in die Reaction waren eingetreten 0,0445 Grm. Br, d. h. 7,6\%. Bei den Versuchen 4. und 5. wurde die Bromwasserstoffsäure durch Wasser ersetzt, der Versuch 6. aber mit trocknem Brom und Benzol ausgeführt Beim Vergleich dieser letzten Versuche mit den übrigen, in Anwesenheit von Bromwasserstoffsäure ausgeführten, erweist sich ein Unterschied zu Gunsten von diesen übrigen. Obgleich dieser Unterschied sehr gering ist, überschreitet er doch die Grenzen eines Versuchsfehlers. Folglich muss man annehmen, dass der Bromirungsprocess des Benzols durch die Gegenwart kleiner Quantitäten von Bromwasserstoffsäure beschleunigt wird; doch ist diese Beschleunigung bei den Bedingungen der Versuche, die von Anfang an die Bildung der Verbindungen zwischen Bromwasserstoffsäure und Brom sehr beschränken, unbedeutend.

Ueber den Einfluss der Chlorwasserstoffsäure sowie einiger Chlor- und Bromsalze auf die Reaction zwischen Brom und Trimethylen werde ich nur kurz berichten, weil alle diese Verbindungen ähnlich der Bromwasserstoffsäure, doch schwächer als diese wirken.

XX. $12 \mathrm{Grm} . \mathrm{Br}$ wurden mit $0,3 \mathrm{Ccm}$. Chlorwasserstoffsäure vom spec. Gew. 1,19, was 0,1134 Grm. HCl entspricht, versetzt. Durch diese Mischung wurden $1750 \mathrm{Ccm}$. Trimethylen geleitet, wobei eine Temperaturerhöhung beobachtet wurde. An Oel wurden 1,52 Grm. gewonnen, dabei hatten sich 0,0642 Grm. $\mathrm{HBr}$ gebildet. Das Resultat dieses Versuchs nähert sich folglich dem Resultat des Versuchs mit Wasser allein. Eine ähnliche Wirkung auf die Reaction zeigt unter sonst gleichen Bedingungen eine bei $18^{\circ}$ gesättigte Kochsalzlösung. Doch in dieser Richtung untersuchte Natriumbromidund Baryumbromidlösungen ergaben höhere Resultate.

XXI. Es wurden in ein Gemisch von $12 \mathrm{Grm}$. Brom und $0,3 \mathrm{Ccm}$. einer bei $18^{\circ}$ gesättigten Natriumbromidlösung $1500 \mathrm{Ccm}$. Trimethylen hindurchgeleitet, dabei 2,75 Grm. Oel und 0,1387 Grm. $\mathrm{HBr}$ gefunden.

XXII. Durch $12 \mathrm{Grm}$. Brom und $0,3 \mathrm{Ccm}$. einer bei $18^{\circ}$ gesättigten Lösung von $\mathrm{BaBr}_{2}$ wurden $1500 \mathrm{Ccm}$. Tri- 
methylen geleitet, dabei 2,07 Grm. Oel und 0,1069 Grm. $\mathrm{HBr}$ gefunden.

Wenn man Trimethylen durch Brom leitet, welches man mit immer grösseren Quantitäten von Bromsalzlösungen verdünnt, so nimmt die Absorptionsgeschwindigkeit rasch ab und die Absorption ist zuletzt nicht mehr bemerkbar; diese Erscheinung wurde auch hinsichtlich der Bromwasserstoffsäure beobachtet. Beim Leiten von Trimethylen durch die Lösung von Brom in 5-6 Volumen gesättigter Natriumbromidlösung bemerkt man keine Bildung von Bromiden in Form des Oeles, obgleich etwas Trimethylen von der Lösung zurückgehalten wird. Leitet man jedoch durch dieselbe Bromlösung Propylen, so wird dieses vollständig absorbirt, wenn man nur das Gas nicht zu schnell hindurchstreichen lässt. Auf diesem Wege kann man die Trennung des Trimethylens vom Propylen bewerkstelligen, was ich bei der Untersuchung der Produkte der Reaction von Brom auf Trimethylen benutzt habe, wie aus dem weiter Folgenden ersichtlich sein wird.

Zur Trennung der Produkte der Reaction von Trimethylen auf Brom $^{1}$ ) habe ich angewandt: 1. Destillation, 2. Durchleiten des aus den Bromiden dargestellten Trimethylens und Propylens durch eine Lösung von Brom in 5-6 Volumen gesättigter Natriumbromidlösung, 3. Einwirkung von festem, mit Wasser angefeuchtetem Permanganat auf das Gemisch von Trimethylen und Propylen. In quantitativer Hinsicht geben alle diese Methoden nur annähernde Resultate.

Die Destillation wurde in allen Fällen zur Trennung der mittleren Portion der Bromide von den niedriger und höher siedenden Produkten angewandt. Bei allen Versuchen siedete die Hauptmasse bei $140^{\circ}-167^{\circ}$. Die niedriger siedenden Portionen gingen immer bei $70^{\circ}-72^{\circ}$ über (Propylbromid). Die höher als $167^{\circ}$ siedenden waren die Produkte der weiteren

1) Das Gemisch der Bromide wurde von dem Brom, durch welches das Trimethylen geleitet worden war, durch Natronlauge getrennt, und zwar immer sofort nach Beendigung des Versuchs, um einer weiteren Einwirkung des Broms auf das in ihm gelöste Trimethylen und die anderen Produkte vorzubeugen. 
284 Gustavson: Ueb.d.Einw.d. Broms auf Trimethylen etc.

Einwirkung von Brom auf Trimethylen- und Propylenbromid. Die mittlere Portion wurde in manchen Fällen noch einmal destillirt, um die Anwesenheit von Propylenbromid in dieser Portion zu beweisen. Ich füge jedoch hinzu, dass eine vollständige Trennung von Propylen- und Trimethylenbromid auf diesem Wege nicht erreicht werden kann, wie ich es schon früher bei der Destillation grosser Quantitäten käuflichen Trimethylenbromids bemerkt habe. Eine Lösung von Brom in 5-6 Volumen gesättigter Natriumbromidlösung kann man, wie schon oben erwähnt, zur Trennung von Trimethylen und Propylen anwenden. Hierbei kann man das Gasometer vermeiden und, ohne die Gase über Wasser aufzufangen, was immer grosse Ungenauigkeiten mit sich führt, sie direct in Bromlösung leiten. Im Falle, dass in den Gasen Propylen vorhanden war, bildet sich in der Bromlösung eine untere Oelschicht, welche man, nachdem alles Propylen in Bromlösung durch einen Luftstrom iubergeführt ist, sammeln, vom Brom trennen und wägen kann.

Die Bestimmung des Trimethylens und Propylens mittelst festem, mit Wasser angefeuchtetem Permanganat wurde von mir schon im vorigen Jahre vorgeschlagen. ${ }^{1}$ ) Die Zersetzung der bei $140^{\circ}-167^{\circ}$ siedenden Portion mittelst Zinkstaub und Alkohol führt man in einem Reagensglase von $5-6 \mathrm{Ccm}$. Inhalt aus, welches mit einem kleinen Rückflusskühler verbunden ist, und fängt das Gas in Messröhren von 50 bis $60 \mathrm{Ccm}$. Inhalt auf. Da die Zusammensetzung des Gases zu Beginn und am Schluss des Versuches nicht die gleiche ist (es entwickelt sich aufangs Propylen und dann Trimethylen), so ist man eben genöthigt, in dem Messrohr das Gas zusammen mit der in dem Apparat befindlichen Luft aufzufangen. Wenn nach dem langen Kochen der Flüssigkeit im Entwicklungsgefäss das Volum des Gases in dern Messrohr nicht mehr zunimmt, liest man dasselbe bei Zimmertemperatur und Atmosphärendruck ab, darauf führt man in das Messrohr von unten ein mit zerkleinertem und vom Wasser bedeckten Permanganat beschicktes Probirgläschen ein, schliesst das Messrohr unter Wasser mit einem eingeschliffenen Stöpsel, lässt es unter

1) Ber. d. Kais. Acad. d. Wissensch. zu Petersburg 1899, Nr. 2. 
öfterem Schütteln eine Stunde lang stehen und liest dann von neuem das Volumen ab, nachdem man unter Wasser oder Salzlösung den Stöpsel entfernt hat. Die Verringerung des Gasvolumens entspricht dem Propylen, das zuletzt abgelesene Volumen nach Abzug des Luftrolumens, welches bei meinen Versuchen 4-4,5 $\mathrm{Ccm}$. betrug, dem Trimethylen. Das Verfahren giebt nur annähernde Resultate und ist für die Bestimmung von kleinen Mengen von Propylen wegen der Löslichkeit von Gasen in Wasser und selbst in Natriumchloridlösung nicht anwendbar. Das Princip aber, auf dem diese Methode begründet ist, d. b. die Beständigkeit des Trimethylens dem Permanganat gegenüber, ist unanfechtbar. Eine sehr verdünnte Lösung von Permanganat, mit einem Ueberschuss von Trimethylen im Rohr eingeschmolzen, wird sogar beim Erhitzen bis $200^{\circ}$ während 7 Stunden nicht entfärbt. Nur nach zweistündigem Erhitzen bei $250^{\circ}$ war in der Röhre mit Trimethylen die Färbung verschwunden, während sie sich in der Proberöhre, welche statt des Trimethylens Luft enthielt, erhalten hatte. Auf diese Weise erweist sich das Trimethylen als im höchsten Grade beständig dem Permanganat gegenüber. Andererseits wird Propylen leicht und vollständig von festem, mit Wasser angefeuchtetem Permanganat oxydirt. In ein Messrohr wurden $50 \mathrm{Ccm}$. Propylen geleitet, sodann ein Probirgläschen mit Wasser und festem Permanganat eingeführt. Beim Schütteln des mit einem eingeschliffenen Stöpsel verschlossenen Messrohres, erhitzte sich dasselbe stark. Nach 20 Minuten liess man in das Messrohr Wasser eintreten: es blieben $3 \mathrm{Ccm}$. Gas zurück, die sich als Luft erwiesen, - sie brannten nicht. Wenn man jedoch anstatt mit Wasser, mit einer gesättigten Kochsalzlösung arbeitet, so vollzieht sich die Oxydation des Propylens langsamer und erfordert eine grosse Menge von Flüssigkeit. ${ }^{1}$ ) Wie ich ermittelt habe, bildet sich bei der Oxydation von Propylen durch festes, mit Wasser angefeuchtetes Permanganat hauptsächlich Essigsäure. Aus $175 \mathrm{Ccm}$. Propylen und 5,4 Grm. Permanganat wurden 0,701 Grm. essigsaures Silber, d. h.

1) Aethylen verhält sich zu festem, mit Wasser angefeuchtetem Permanganat ganz analog dem Propylen. 
286 Gustavson: Ueb.d. Einw.d. Broms auf Trimethylen etc.

0,25 Grm. Essigsäure gewonnen. Wenn sich bei der Oxydation ausschliesslich Essigsäure gebildet hätte, so hätte die angewandte Menge Propylen 0,398 Grm. ergeben müssen.

Die Produkte der Reaction von Brom auf Trimethylen bei verschiedenen Bedingungen wurden durch folgende Versuche ermittelt.

XXIII. Mehrere Glasgasometer, im Ganzen von 13 Liter Inhalt, wurden mit Trimethylen gefüllt (wie immer war dasselbe mittelst Durchleitens durch Brom gereinizt), so dass fast alles Wasser vom Gase verdrängt war. In die Gasometer wurde sodann eine Quantität Brom eingefübrt, die etwas grösser war als die Theorie forderte $\left(\mathrm{C}_{3} \mathrm{H}_{6}: \mathrm{Br}_{2}\right)$. Nach Verlauf von $3 \mathrm{Mal} 24$ Stunden im Dunkeln bei einer Temperatur von $15^{\circ}-18^{\circ}$ wurde ungefähr die Hälfte der theoretischen Menge Oel erhalten. Von diesem Oel wurden $40 \mathrm{Grm}$. einer wiederholten Destillation unterworfen: a) Als niedrigst siedendes Produkt, $70^{\circ}-72^{\circ}$, erwies sich eine $65,54 \% \mathrm{Br}$ enthaltende Substanz (0,1277 Grm. gaben 0,1967 Grm. AgBr). Propylbromid enthält $65,03 \% \mathrm{Br}$. Hiervon wurden jedoch nur ungefähr 0,5 Grm. gewonnen. b) Die folgende Portion siedete bei $139^{\circ}-142^{\circ}$, ungefähr $3 \mathrm{Grm}$., 0,322 Grm. gaben 0,5965 Grm. $\mathrm{AgBr}$, d. h. $78,82 \% \mathrm{Br} ; \mathrm{C}_{3} \mathrm{H}_{8} \mathrm{Br}_{2}$ enthält $79,20 \%$ Br. Die Bestimmung des spec. Gew. ergab bei $\frac{18^{0}}{4^{0}} 1,9346$. Nach Zander beträgt das spec. Gew. des Propylenbromids bei $\frac{18^{\circ}}{0^{\circ}}$ 1,9307. Diese Portion enthielt folglich Propylenbromid. c) Die Portionen, die bei $145^{\circ}-159^{\circ}$ (2,5 Grm.) und bei $159^{\circ}$ bis $167^{\circ}$ (22 Grm.) siedeten, wurden zusammen in Alkohol gelöst und mit einer zur vollständigen Zersetzung ungenügenden Menge Zinkstaub bearbeitet. Nach der Entwicklung von 1 Liter Gas wurde die Reaction unterbrochen. Das aus dem Reste ausgeschiedene Trimethylenbromid siedete bei $166^{\circ}$ bei $780 \mathrm{Mm}$. Druck und sein spec. Gew. betrug 1,9869 bei $\frac{17,25^{\circ}}{4^{0}}$. Das gesammelte Gas (1 Liter) wurde durch Brom geleitet, welches mit 5 Thln. gesättigter Natriumbromidlösung verdünnt war; hierbei bildeten sich 1,05 Grm. Oel, welches bei $140^{\circ}$ bis $145^{\circ}$ siedete. Dieses Oel wurde mittelst Zinkstaub und Alkohol zersetzt und das erhaltene Gas der Einwirkung von 
festem Permanganat mit Wasser unterworfen, wobei keine brennbaren Gase zurückblieben. d) Von der höher als bei $167^{\circ}$ siedenden Portion wurden 9,7 Grm. erhalten. Der bei $185^{\circ}-190^{\circ}$ siedende Theil ergab bei der Analyse $82,3 \% \mathrm{Br}$. Folglich enthält diese Portion Produkte, die reicher an Brom sind als $\mathrm{C}_{3} \mathrm{~B}_{8} \mathrm{Br}_{2}$. Im Wasser wurde Bromwasserstoff gefunden, dessen Quantität aber nicht bestimmt wurde. Die angeführten Versuche beweisen, dass Trimethylen, welches kein Propylen enthält, trotzdem bei der Einwirkung von Brom neben anderen Produkten bis zu 10\% Propylenbromid bildet.

XXIV. In eine Glasflasche von $3280 \mathrm{Ccm}$. Inhalt wurde eine zugeschmolzene, mit 20,86 Grm. Brom und 0,5 $\mathrm{Ccm}$. Wasser beschickte Röhre gethan. Aus der Glasflasche wurde die Luft bis auf einen Druck von $80 \mathrm{Mm}$. ausgepumpt, trocknes Trimethylen unter $765 \mathrm{Mm}$. Druck und bei $18^{\circ}$ hineingeleitet und dann die Röhre mit Brom zerbrochen. Hieraus kann man berechnen, dass anstatt 79,2 Grm. Br auf 20,8 Grm. Trimethylen $\left(\mathrm{C}_{3} \mathrm{~B}_{8}: \mathrm{Br}_{2}\right) 80,1 \mathrm{Grm}$. $\mathrm{Br}$ auf $19,9 \mathrm{Grm} . \mathrm{C}_{3} \mathrm{H}_{6}$ genommen worden sind. Nach 24 Stunden (bei $14^{\circ}-18^{\circ}$, im Dunkeln) wurde die Reaction unterbrochen und 7,85 Grm. Oel ausgeschieden. Hierbei hatten sich 0,289 Grm. HBr gebildet. Aus den Bromiden wurde eine bei $70^{\circ}-73^{\circ}$ siedende Portion abgeschieden. 0,091 Grm. Substanz gaben 0,1392 Grm. AgBr, d. h. $65,08 \% \mathrm{Br} ; \mathrm{C}_{3} \mathrm{~B}_{7} \mathrm{Br}$ enthält $65,03 \% \mathrm{Br}$. In den Gasen aus der bei $140^{\circ}-167^{\circ}$ siedenden Portion wurden durch Einwirkung von festem Permanganat über Wasser 10,2\% Propylen ermittelt.

XXV. In eine Glasflasche von $3280 \mathrm{Ccm}$. Inhalt wurde eine zugeschmolzene Röhre mit 19,85 Grm. Br gethan, die Luft aus der Flasche bis auf $75 \mathrm{Mm}$. ausgepumpt, trocknes Trimethylen unter $750 \mathrm{Mm}$. Druck bei $18^{\circ}$ eingeleitet und die Röhre mit Brom zerbrochen. Nach 4 tägigem Stehen im Dunkeln bei einer Temperatur von $15^{\circ}-18^{\circ}$ wurden 6,55 Grm. Bromide gewonnen. Die bei $70^{\circ}-\mathbf{7 4}^{\circ}$ siedende Portion gab aus 0,0897 Grm. 0,1392 Grm. AgBr, d. b. 66,09\% Br. Die Brombestimmung in der bei $190^{\circ}$ siedenden Portion ergab aus $0,1723 \mathrm{Grm}$. 0,3316 Grm. $\mathrm{AgBr}$, d. h. $81,88 \%$ Br. Von der bei $140^{\circ}-167^{\circ}$ siedenden Portion wurden 4,1 Grm. erhalten. Die ganze Portion wurde in Gas umgesetzt, welches 
288 Gustavson: Ueb.d. Einw.d. Broms auf Trimethylen etc.

beim Durchleiten durch eine Lösung von Brom in $5 \mathrm{Vol}$. Natriumbromidlösung 0,63 Grm. Propylenbromid ergab. Das hieraus entwickelte Propylen wurde von festem Permanganat in Gegenwart einer nur geringen Menge Wasser vollständig absorbirt: es blieb nur ein wenig Luft zurück. Folglich bilden sich bei der Einwirkung von trocknem Trimethylen auf trocknes Brom auch ungefähr $10 \%$ Propylenbromid, wenn man es auf das ganze Gemisch der Bromide berechnet, und ungefähr $15 \%$, auf die bei $140^{\circ}-167^{\circ}$ siedende Portion berechnet.

XXVI. Aus 5,6075 Grm. der bei $140^{\circ}-167^{\circ}$ siedenden Portion, welche aus dem beim Durchleiten von Trimethylen durch $24 \mathrm{Grm}$. Brom und 0,6 Ccm. $\mathrm{HBr}+5 \mathrm{H}_{2} \mathrm{O}$ (s. Vers. XII) erhaltenen Oele gewonnen war, wurde mittelst Zinkstaub und Alkohol Gas entwickelt. Durch eine Bromlösung in 5 Vol. Natriumbromidlösung hindurchgeleitet, ergab dasselbe 0,743 Grm. Oel, d. h. 13,2\% Propylen für die bei $140^{\circ}-167^{\circ}$ siedende Portion.

XXVII. Aus den Bromiden, welche bei der Einwirkung von Trimethylen auf $12 \mathrm{Grm}$. Brom und $0,3 \mathrm{Ccm}$. $\mathrm{HBr}+5 \mathrm{H}_{2} \mathrm{O}$ gewonnen waren, wurde eine bei $140^{\circ}-167^{\circ}$ siedende Portion ausfractionirt. In dem aus dieser Portion entwickelten und über einer Kochsalzlösung aufgefangenen Gase wurden mit festem Permanganat 14,7\% Propylen ermittelt.

XXVIII. Aus 2,65 Grm. Oel, welches beim Durchleiten von $1750 \mathrm{Ccm}$. Trimethylen durch $12 \mathrm{Grm}$. Brom und $3 \mathrm{Ccm}$. $\mathrm{HBr}+5 \mathrm{H}_{2} \mathrm{O}$ (Vers. VIII) gewonnen war, wurden durch Fractionirung ungefähr $10 \%$ einer bei $70^{\circ}-72^{\circ}$ siedenden Portion abgeschieden. 0,2263 Grm. dieser Portion gaben 0,3447 Grm. $\mathrm{AgBr}$, d. h. $64,76 \% \mathrm{Br}$ gegen $65,03 \% \mathrm{Br}$ des Propylbromids. Dieser Versuch zeigt, dass mit grösseren, zur Reaction verwandten Mengen Bromwasserstoffsäure auch die Menge von Propylbromid zunimmt, d. h. das letztere bildet sich mit Hülfe der Bromwasserstoffsäure aus dem Trimethylen. ${ }^{1}$ )

1) Die Natur des Propylbromids wurde auf Grund meiner früheren Versuche dadurch bewiesen, dass es mit einer sehr geringen Menge Aluminiumbromid in eine Röhre eingeschmolzen und 24 Stunden lang stehen gelassen wurde. Nach Ablauf dieser Zeit wurde seine Umwandlung in das um $10^{\circ}$ niedriger siedende Isopropylbromid constatirt. 
Die angeführten Versuche beweisen, dass von Propylen freies Trimethylen bei seiner Reaction mit Brom im Dunkeln oder bei zerstreutem Tageslicht in Gegenwart von Wasser, oder in trocknem Zustande, oder endlich in Gegenwart von Bromwasserstoffsäure ausser anderen Produkten immer auch Propylenbromid bildet. Neben Propylenbromid wurde bei allen Versuchen auch Propylbromid gefunden. Die Entstehung des letzteren erklärt sich durch die Einwirkung von Bromwasserstoff auf Trimethylen. ${ }^{1}$ ) Der Bromwasserstoff jedoch

1) Nicht durch die Einwirkung von Brom auf Propan, wie B. Menschutkin und A. Wolk off behaupten (J. d. Russ. Phys. Ch. Ges. 32, 118; Chem. Centrbl. 1900, 2, 42), welche auch die Entstehung von Propylenbromid aus dem von Propylen befreiten Trimethylen und Brom auf Rechnung des Propans setzen. Natürlich musste man bei solcher Behauptung in erster Linie die Anwesenheit des Propans in dem nach meiner Methode dargestellten Trimethylen beweisen. B. Menschutkin und A. W ol k of glauben das darzuthun, indem sie bei derVerbrennungsanalyse unbestimm ter Mengen von Trimethylen statt des theoretischen Verhältnisses zwischen Wasserstoff und Kohlenstoff (2:1) etwas mehr Wasserstoff (2,04:1; 2,09:1) gefunden haben. Der von B. Menschutkin und A. Wolk off gefundene Ueberschuss an Wasserstoff ist aber so unbedeutend (im Mittel weniger als $0,5 \%$ ), dass daraus überhaupt kein Schluss auf Unreinheit von Trimethylen gezogen werden kann, umsomehr, als die Analyse des nach derselben Methode dargestellten Trimethylens, von Tornoë (Ber.21, 1282) ausgeführt, ganz der Theorie entspricht. Andererseits machte die totale Absorption von Trimethylen durch Brom in Gegenwart von Bromwasserstoffsäure (Vers. XIII und XIV) im Falle der Anwesenheit von Propan die Annahme nothwendig, dass auch dieses Gas vom Brom und Bromwasserstoffsäure vollständig absorbirt wurde. Nun habe ich gefunden, dass Propan sich sebr indifferent gegen Brom in Gegenwart von Bromwasserstoffsäure wie auch gegen Brom allein verhält. Beim Durchleiten von 1 Lit. Propan durch $12 \mathrm{Grm} . \mathrm{Br}+0,3 \mathrm{Ccm}$. $\mathrm{HBr} 5 \mathrm{H}_{2} \mathrm{O}$ während 1 Stunde war die Menge des gebildeten Oeles so gering, dass man es nicht sammeln und wägen konnte. Nach diesen Versuchen sollte man erwarten, dass in dem Falle der Anwesenheit von Propan in nach meiner Methode dargestelltem Trimethylen das erstere sich grösstentheils in durchgeleiteten Gasen concentriren muss, was gar nicht bemerkt wurde. Um die Frage endgültig zu lösen, habe ich absichtlich 2 Lit. Trimethylen $60 \mathrm{Ccm}$. Propan beigemischt, und dieses $3 \%$ Propan enthaltende Gasgemisch wurde durch $12 \mathrm{Grm}$. Brom, dann durch Natronlauge geleitet und im Gasometer über Wasser gesammelt. $400 \mathrm{Ccm}$. davon wurden während $30 \mathrm{Min}$. durch $40 \mathrm{Grm}$. Schwefelsăure hindurchgeleitet. Im Messrohre waren $25 \mathrm{Ccm}$. Gas gesammelt, welches mit heller Flamme brannte. Aus der totalen Absorption des nach meiner 
290 Gustarson: Ueb.d.Einw.d. Broms auf Trimethylen etc.

bildet sich bei der zwischen Brom und Trimethylen stattfindenden Reaction selbst, wie schon oben bewiesen wurde. Dass bei der Einwirkung von Bromwasserstoff auf Trimethylen Propylbromid entsteht, geht aus folgenden Versuchen hervor:

XXIX. Aus einer Glasflasche von $3280 \mathrm{Ccm}$. Inhalt wurde die Luft bis auf einen Druck von $120 \mathrm{Mm}$. ausgepumpt und trocknes Trimethylen eingeleitet. Darauf wurden in die Glasflasche rasch $10 \mathrm{Ccm}$. rauchender, $61,45 \% \mathrm{HBr}$ enthaltender Bromwasserstoffsäure gegossen und die verschlossene Flasche 5 Tage lang stehen gelassen. Es hatten sich 5,5 Grm. Propylbromid gebildet, welches bei $71^{\circ}$ überging.

XXX. Fin Liter Trimethylen wurde erst durch $2 \mathrm{Ccm}$. $\mathrm{HBr} 5 \mathrm{H}_{2} \mathrm{O}$ und dann durch Wasser geleitet. Die Titration des Wassers und der Säure ergab 1,356 Grm. HBr. Vor dem Versuch enthielten die $2 \mathrm{Ccm}$. Säure $1,454 \mathrm{Grm}$. HBr. Folglich hatten sich $0,098 \mathrm{Grm}$. HBr mit Trimethylen verbunden.

Aus den angeführten Versuchen geht hervor, dass das Trimethylen der Einwirkung von Bromwasserstoffsäure leicht zugänglich ist, sogar wenn die letztere sich in Form des Hydrates $\mathrm{HBr} 5 \mathrm{H}_{2} \mathrm{O}$ befindet. Bei der Reaction von Brom mit Trimethylen bleibt das entstandene Propylbromid in Berührung mit den reagirenden Körpern bei gleichzeitiger Anwesenheit von Brom, und es liegt am nächsten, die Bildung von Propylenbromid bei dieser Reaction durch die weitere Einwirkung von Brom auf das Propylbromid zu erklären. Ich muss jedoch bemerken, dass Versuche über Einwirkung von Brom auf Propylbromid, welche in unserem Laboratorium von Frl. N. Petruschewsky und Frl. A. Zeidler ausgeführt wurden, eine bemerkenswerthe Indifferenz des letzteren

Methode mittelst 75 proc. Alkohol und Zinkstaub dargestellten Trimethylens durch Brom und Bromwasserstoffsäure sowie durch Schwefelsäure geht also hervor, dass Trimethylen kein Propan enthält, und da aus Trimethylen, mit welehem ich arbeitete, auch Propylen durch Brom entfernt war, so folgt weiter, dass Propylenbromid wie auch Propylbromid, welche in den Produkten der Reaction des Broms auf Trimethylen gefunden waren, aus Trimethylen selbst und nicht aus dessen nicht existirenden Beimengungen gebildet werden können. 
dem Brom gegenüber ergaben. 0,6722 Grm. Propylbromid wurden mit 0,6589 Grm. trocknen Broms in eine Röhre eingeschmolzen; nach Verlauf von 24 Stunden, bei $15^{\circ}-20^{\circ}$, ergab die Brombestimmung mittelst Jodkalium eine Verringerung der Menge des Broms von nur 1\%. Ein anderer Versuch mit 0,6156 Grm. Propylbromid und 0,3632 Grm. Brom ergab unter denselben Bedingungen eine Verminderung von $0,14 \%$. Bemerkbarer vollzieht sich die Bromirung in Gegenwart von Wasser, wenn man das Gemisch längere Zeit stehen lässt, wie ein von mir ausgefuhrter Versuch zeigt.

XXXI. Es wurden 10 Grm. Brom, 5 Grm. Propylbromid und $0,3 \mathrm{Ccm}$. Wasser bei Zimmertemperatur 21 Tage lang stehen gelassen und nach dieser Zeit 0,1313 Grm. $\mathrm{HBr}$ gefunden. Nachdem das Propylbromid abdestillirt war, wurde der Rest mit Zinkstaub und Alkohol zersetzt: es wurden $22 \mathrm{Ccm}$. Gas erhalten. Bei Einwirkung von festem Permanganat und Wasser fand eine Verringerung des Gasvolumens um 15,4 Ccm. statt.

Die Versuche zeigen demnach, dass sich die Reaction zwischen Brom und Propylbromid sehr langsam vollzieht. Natürlich kann man in Fällen, wie der betrachtete, Ergebnisse der Untersuchung von einzelnen Reactionen nicht direct zur Feststellung des Verlaufes eines complicirten Processes verwenden. Bei dem letzteren kann die Sache anders verlaufen, als wir annehmen, indem wir uns an eine schematische Vorstellung über die Reactionsfolge halten. Einerseits muss man zugeben, dass bei der Einwirkung des Broms auf Trimethylen das sich unter Einfluss von Bromwasserstoff bildende Propylbromid kaum im Entstehungszustand mit Brom reagiren kann; andererseits sind überhaupt die physikalischen Bedingungen, welche in Folge der gleichzeitig verlaufenden Reactionen entstehen, nicht für einzelne absichtlich anzustellende Reactionen einzuhalten. Wie dem auch sei, so entsteht doch bei der Reaction zwischen Brom und Trimethylen Propylbromid, und aus demselben kann Propylenbromid entstehen. Was die früher von mir ausgesprochene Ansicht über die Betheiligung von Bromtrimethylen an der Bildung von Propylenbromid anbetrifft, so hat sich diese Annahme bei der 
292 Gustavs on: Ueb. d. Finw.d. Broms auf Trimethylen etc. gegenwärtigen Untersuchung nicht bestätigt, da unter den Produkten der Reaction kein bromirtes Trimethylen gefunden wurde.

Ich gehe jetzt $z u$ der Einwirkung von Brom auf Trimethylen in Gegenwart von kleinen Mengen Aluminiumbromid über. Wenn man zum Brom eine sehr geringe Menge Aluminium hinzufügt, z. B. so viel, dass das sich bildende Aluminiumbromid ungefähr $1 \%$ des genommenen Broms beträgt, und durch die Lösung trocknes Trimethylen leitet, so überrascht die Reaction durch ihre Intensität. Es ist eine Abkühlung bis $0^{0}$ nöthig, sonst erleiden die sich bildenden Produkte eine weitere Veränderung, die von reichlicher Bromwasserstoffentwicklung begleitet ist, wobei die Flüssigkeit sich schwarz färbt. Sogar bei einem sehr rasch durchstreichenden Gasstrome, z. B. ein Liter in 10-15 Minuten, fand, so lange noch Brom vorhanden war, vollständige Absorption statt. Es ist jedoch bemerkt worden, dass es nicht rathsam ist, mit dem Durchleiten des Trimethylens über das Verschwinden des Broms hinaus fortzufahren: manchmal färbt sich die Flüssigkeit auch bei Abküblung schwarz.

XXXII. Durch 25 Grm. Brom, welche mit 0,03 Grm. Aluminium versetzt waren, wurden 4 Lit. Trimethylen bei $0^{\circ}$ geleitet. Die Reaction wurde nicht ganz zu Ende geführt und $23 \mathrm{Grm}$. Bromide erhalten. Bei der Destillation wurde ungefähr $1 \mathrm{Grm}$. einer bei $59^{\circ}-60^{\circ}$ siedenden Portion ausgeschieden: 0,186 Grm. gaben 0,2821 Grm. AgBr, d. h. $64,53 \%$ Br gegen $65,03 \%$ des Isopropylbromids. Ein bei $70^{\circ}$ siedendes Destillat war nicht vorhanden. Die Hauptmasse siedete bei $140^{\circ}-167^{\circ}$. In dem aus dieser Portion mittelst Zinkstaub und Alkohol entwickelten Gase wurde aus der Verringerung des Gasvolumens bei Einwirkung von Permanganat Propylen bestimmt. a) $32 \mathrm{Ccm}$. verringerten sich bis auf $15 \mathrm{Ccm}$; da sich in den Gasen $4 \mathrm{Ccm}$. Luft befanden, so betrug die Propylenmenge $53,6 \%$ b) $37,8 \mathrm{Ccm}$. verringerten sich bis auf 18,3 Ccm.; die Propylenmenge betrug (ohne $4 \mathrm{Ccm}$. Luft) $54,1 \%$, c) $35 \mathrm{Ccm}$. verringerten sich bis auf $16,3 \mathrm{Ccm}$.; die Propylenmenge betrug (ohne $4 \mathrm{Ccm}$. Luft) 52,6\%. Bei 
Gustarson: Ueb.d. Einw.d. Broms auf Trimethylen etc. 293

der letzten Bestimmung wurde das Gas über Wasser aufgefangen, bei den ersten beiden über einer Natriumchloridlösung.

XXXIII. Aus einer Glasflasche von 2 Lit. Inhalt wurde die Luft ausgepumpt und trocknes Trimethylen hineingeleitet. Darauf wurden $10 \mathrm{Grm}$. Brom mit einigen in ihm gelösten Aluminiumspähnen und 10 Minuten danach Wasser in die Flasche gegossen. Es wurden 9,3 Grm. Oel erhalten. In $38 \mathrm{Ccm}$. Gas aus der bei $140^{\circ}-167^{\circ}$ siedenden Portion (7 Grm.) wurden mittelst Permanganat 19,7 Ccm. Propylen bestimmt, d. h. $51,84 \%$.

Aus den angeführten Versuchen folgt, dass Aluminiumbromid die Vereinigung von Brom mit Trimethylen stark beschleunigt. $\mathrm{Ob}$ es hierbei das Trimethylen schon zum Theil vor der Vereinigung mit Brom in Propylen umwandelt, wird durch besondere Versuche ermittelt werden; dass jedoch Trimethylenbromid unter Einwirkung von Aluminiumbromid sich leicht in Propylenbromid umwandelt, ist aus meinen früheren Arbeiten bekannt. Interessant erscheint jedenfalls in den angeführten Versuchen die Geschwindigkeit, mit der sich Trimethylen in Propylenbromid umwandelt. Sie übertrifft bei weitem die Umwandlungsgeschwindigkeit, welche man aus den Versuchen über die Einwirkung von Aluminiumbromid auf Trimethylenbromid ermitteln kann. Wenn man z. B. Trimethylenbromid mit $1 \%$ Aluminiumbromid in eine Röhre einschmilzt und nach 2 Stunden in dem mittelst Zinkstaub und Alkohol entwickelten Gase Propylen bestimmt, so beträgt die Menge desselben ungefähr 10\% gegen $90 \%$ Trimethylen. ${ }^{\text {) }}$ Das bei der Reaction von Brom auf Trimethylen in Gegenwart von Aluminiumbromid gefundene Isopropylbromid ist

1) In Anbetracht der Leichtigkeit, mit welcher sich Propylenbromid bei der Einwirkung von Brom und Aluminiumbromid auf Trimethylen bildet, wurde die Einwirkung dieser Körper auf Aethylen untersucht, ob nicht Aethylenbromid entstehen würde. 3 Lit. Aethylen wurden dureh $20 \mathrm{Grm}$. Brom und $2 \mathrm{Grm}$. Aluminiumbromid geleitet. Es wurden 22 Grm. Oel gewonnen, welches vollständig bei $130^{\circ}-131^{\circ}$ überging. Aethylidenbromid bildet sich also nicht. Ich bemerke bierbei noch, dass sich bei der Einwirkung von Aluminiumbromid auf Propylenbromid auch kein $\mathrm{CH}_{3} \cdot \mathrm{CBr}_{2} \cdot \mathrm{CH}_{3}$ bildet. 
294 Gustavson: Ueb.d. Einw. d. Broms anf Trimethylen etc.

natürlich das Produkt einer isomeren Umlagerung des Propylbromids unter dem Einfluss von Aluminiumbromid.

Chloreisen beeinflusst die Reaction von Brom auf Trimethylen in ähnlicher Weise, wie das Aluminiumbromid. Die Wirkung ron Jod und Chlorzink ist jedoch bedeutend schwächer. Ueber diese Reactionen, wie über die fördernde Wirkung ron Knochenkohle auf die Verbindung von Brom mit Trimethylen wird in den nächsten Abhandlungen berichtet werden.

Sonnenlicht unterscheidet sich von allen anderen Erregern dadurch, dass unter dessen Einfluss aus Trimethylen und Brom fast gar kein Propylenbromid sich bildet.

XXXIV. Aus einer Glasflasche von 3 Lit. Inhalt wurde die Luft bis auf $150 \mathrm{Mm}$. Druck ausgepumpt und trocknes Trimethylen hineingeleitet. Dann wurde 12,2 Grm. Brom hineingebracht und die Flasche dem Einfluss des directen Sonnenlichts im April bei ganz hellem Himmel ausgesetzt. Nach einer $1 / 2$ Stunde war Brom fast ganz verschwunden und der Versuch war unterbrochen. An Oel wurden $11 \mathrm{Grm}$. gewonnen und im Waschwasser 0,107 Grm. $\mathrm{HBr}$ gefunden. Beim Destilliren des Oeles ging alles bei $163^{\circ}-166^{\circ}$ über. Bei der qualitativen Untersuchung des mittelst Zinkstaub und Alkohol aus dem Oel gewonnenen Gases auf Propylen wurde nur sehr langsame Entfärbung von stark verdünnter Permanganatlösung bemerkt. Der Versuch, Propylen quantitativ nach der Permanganatmethode zu bestimmen, gelingt nicht, weil die Volumverminderungen des Gases zu gering waren. Beim Leiten des Gases durch die Lösung des Broms in Natriumbromid wurden ebenfalls nur unbestimmbare Spuren an Oel bemerkt. Bei der Einwirkung des Sonnenlichts auf Trimethylen und Brom in Gegenwart von Wasser wurden die gleichen Resultate erhalten. In beiden Fällen konnte ich nicht durch Destillation Propylbromid und Propylenbromid entdecken.

Unter dem Einfluss des Sonnenlichts erhält man also Trimethylenbromid aus dessen Componenten am reinsten, was ich schon in meiner ersten Abhandlung über Trimethylen ${ }^{1)}$

1) Dies. Journ. [2] 36, 300 . 
Maksimowitsch: Ueb. den fünfatomigen Alkohol etc. 295

bemerkt habe. Unter allen anderen bis jetzt angewendeten Bedingungen ist das Hauptprodukt der Reaction immer mit Propylbromid und ansehnlichen Mengen von Propylenbromid gemengt. Die Bildung dieser Verbindungen ist durch Sprengung des Trimethylens hervorgerufen, welches nicht nur unter dem Einfluss des Broms, sondern auch unter dèm Einfluss des bei der Reaction sich bildenden Bromwasserstoffs gesprengt wird. Es bildet sich dabei Propylbromid, aus welchem Propylenbromid entstehen kann. Diese Verbindungen sind also aus Trimethylen entstanden, und Propan, welches nicht zugegen ist, spielt, entgegen der Behauptung von B. Menschatkin und A. Wolk off, dabei keine Rolle.

Laboratorium der Frauenhochschule zu St. Petersburg, 30. Mai 1900.

\section{Untersuchungen ans dem chemischen Laboratorium von Prof. Alexander Saytzeff zu Kasan.}
79. Ueber den fünfatomigen Alkohol aus Methyldiallylcarbinol; von

\section{Stefan Maksimowitsch.}

Bei der Oxydation des im hiesigen Laboratorium von B. Sorokin ${ }^{1}$ ) synthetisch hergestellten Methyldiallylcarbinols mittelst Chromsäuregemisch und mit Kaliumpermanganat wurden im ersten Falle ${ }^{2}$ ) Kohlensäure and Essigsäure, im zweiten Falle $^{3}$ ) $\beta$-Methyloxyglutarsäure, Oxalsäure und Kohlensäure erhalten. Auf Grund der Arbeiten von Kekulé über die Oxydation der Fumar- und Maleïnsäure, ferner der im Laboratorium von Prof. A. Saytzeff ausgeführten Untersuchungen über die Oxydation der isomeren Oelsäuren und der mit

1) Ann. Chem. 185, 169.

2) Das. 185, 173.

s) Dies. Journ. [2] 23, 276. 